\title{
Changes in cortical plasticity across the lifespan
}

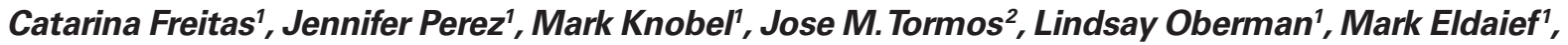 Shahid Bashir ${ }^{1}$, Marine Vernet ${ }^{1}$, Cleofé Peña-Gómez ${ }^{3}$ and Alvaro Pascual-Leone ${ }^{1,2 *}$}

\author{
Berenson-Allen Center for Noninvasive Brain Stimulation, Division of Cognitive Neurology, Department of Neurology, Beth Israel Deaconess Medical Center, Harvard \\ Medical School, Boston, MA, USA. \\ 2 Institut Universitari de Neurorehabilitació Guttmann, Universidad Autónoma de Barcelona, Badalona, Spain \\ 3 Facultat de Medicina, Departament de Psiquiatria i Psicobiologia Clínica, Universitat de Barcelona, Barcelona, Spain
}

\section{Edited by:}

Isidro Ferrer, University of Barcelona,

Spain

\section{Reviewed by:}

Luis Francisco Gonzalez-Cuyar, University of Maryland School of Medicine, USA

Alejandra Alonso, College of Staten Island, USA

\section{*Correspondence:}

Alvaro Pascual-Leone, Berenson-Allen

Center for Noninvasive Brain

Stimulation, Beth Israel Deaconess

Medical Center, 330 Brookline Avenue,

Boston, MA 02215, USA.

e-mail:apleone@bidmc.harvard.edu
Deterioration of motor and cognitive performance with advancing age is well documented, but its cause remains unknown. Animal studies dating back to the late 1970 s reveal that ageassociated neurocognitive changes are linked to age-dependent changes in synaptic plasticity, including alterations of long-term potentiation and depression (LTP and LTD). Non-invasive brain stimulation techniques enable measurement of LTP- and LTD-like mechanisms of plasticity, in vivo, in humans, and may thus provide valuable insights. We examined the effects of a 40-s train of continuous theta-burst stimulation (cTBS) to the motor cortex (600 stimuli, three pulses at $50 \mathrm{~Hz}$ applied at a frequency of $5 \mathrm{~Hz}$ ) on cortico-spinal excitability as measured by the motor evoked potentials (MEPs) induced by single-pulse transcranial magnetic stimulation before and after cTBS in the contralateral first dorsal interosseus muscle. Thirty-six healthy individuals aged 19-81 years old were studied in two sites (Boston, USA and Barcelona, Spain). The findings did not differ across study sites. We found that advancing age is negatively correlated with the duration of the effect of CTBS $(r=-0.367 ; p=0.028)$ and the overall amount of corticomotor suppression induced by cTBS ( $r=-0.478 ; p=0.003$ ), and positively correlated with the maximal suppression of amplitude on motor evoked responses in the target muscle $(r=0.420 ; p=0.011)$. We performed magnetic resonance imaging (MRI)-based individual morphometric analysis in a subset of subjects to demonstrate that these findings are not explained by age-related brain atrophy or differences in scalp-to-brain distance that could have affected the TBS effects. Our findings provide empirical evidence that the mechanisms of cortical plasticity area are altered with aging and their efficiency decreases across the human lifespan. This may critically contribute to motor and possibly cognitive decline.

Keywords: cortical plasticity, aging, motor cortex, transcranial magnetic stimulation, continuous theta-burst stimulation, long-term depression

\section{INTRODUCTION}

The average age of the world's population is increasing at an unprecedented rate. Demographic projections suggest that the number of people aged over 65 years will nearly triple by 2050, accounting for $14 \%$ of the world and $20 \%$ of the US population (Lutz et al., 2008). Aging is the major risk factor for Alzheimer's disease (AD) and is associated with a more or less pronounced decline of many cognitive, motor, and other brain functions (e.g., Deary et al., 2009). This has been behaviorally well-established for decades (e.g., Feier and Gerstman, 1980), but it is unclear that functional decline is an obligatory consequence of aging and the underlying neurophysiologic correlates remain elusive. Neuroimaging studies have revealed various correlates of age-related changes in brain activation (e.g., Cabeza et al., 2002) and large-scale brain functional networks (e.g., Andrews-Hanna et al., 2007; Damoiseaux et al., 2008; Sambataro et al., 2010; Grady et al., 2010; Wang et al., 2010). However, what may trigger such changes remains to be understood. Despite important advances in medicine and very large financial expenditures, morbidity, and disability across aging remain high. Therefore, a greater understanding of the neurobiology underlying age-related decline of function is urgently needed, as it may lead to novel interventions to prevent cognitive decline and minimize the consequences of $\mathrm{AD}$. Unless we work to reduce age-related functional decline and the impact of $\mathrm{AD}$, we will succumb to the staggering personal, social, and economic burden of aging and dementia.

Animal studies building on pioneering work from Barnes (1979) in the late 1970s have demonstrated an age-associated decline in synaptic plasticity in specific brain regions that correlates with neurocognitive impairments. In aged rodents, thresholds for induction of hippocampal long-term potentiation (LTP) and long-term depression (LTD) appear to increase and decrease, respectively (Rosenzweig and Barnes, 2003). Once induced, LTP decays faster in older rats, and this appears to be associated with a greater degree of forgetfulness (Barnes and McNaughton, 1980; Kelly et al., 2006). Moreover, deficits in the balance between LTP and LTD result in impaired learning and memory (Larson et al., 1986; Roman et al., 1987; Bliss et al., 2003). If translated into humans, similar assessments of plasticity mechanisms may shed light into the neurobiological mechanisms underlying normative aging and, ultimately, neurodegenerative processes.

A recently developed protocol for transcranial magnetic stimulation (TMS) can provide information about the integrity of human plasticity in specific, well-defined cortical regions 
(Huang et al., 2005; Oberman et al., 2010). Specifically, continuous theta-burst stimulation (cTBS) can produce sustained effects ( $\sim 60 \mathrm{~min})$ on the reactivity of the underlying neural tissue, as measured by the response to single-pulse TMS before versus after TBS. Animal and human studies reveal that such modulatory effects of TBS on cortical reactivity reflect LTP- and LTD-like mechanisms (Cárdenas-Morales et al., 2010). We use the term plasticity to refer to such LTP- and LTD-like processes in humans as captured by the TBS paradigm (Oberman et al., 2010). We hypothesize that such defined plasticity mechanisms may become increasingly less efficient with advancing age. To test our hypothesis, we conducted a series of studies in healthy adult volunteers across the age-range to assess cortical plasticity in the motor cortex using TMS measures before and serially following neuromodulation by cTBS.

\section{MATERIALS AND METHODS PARTICIPANTS}

Thirty-six healthy volunteers were studied in Boston (MA, USA) and Barcelona (Spain) following a similar experimental protocol. All subjects were right-handed (according to the Oldfield Questionnaire; Oldfield, 1971) and ranged in age from 19 to 81 years. All had a normal mental status as assessed by the MiniMental State Examination (Folstein et al., 1975; $\geq 29$ ) and a normal neurological exam. None had a history of alcohol or substance abuse, unstable medical conditions, previous history of psychiatric illness, learning disability, or neurological disorders (epilepsy, traumatic brain injury, brain tumor, stroke, or neurodegenerative disorders). All gave their written informed consent to the study, which had been approved by the local Institutional Review Boards.

\section{EXPERIMENTAL PARADIGM}

Cortico-spinal motor plasticity was investigated with the use of single-pulse TMS performed at baseline and at several time-points following the delivery of cTBS (Huang et al., 2005, 2008). Singlepulse TMS was performed with a MagPro stimulator (MagVenture, Denmark) equipped with a 75 -mm figure-of-eight (F8) static cooled coil or, in six of our subjects (below), with the Navigated Brain Stimulation (NBS) system (Nexstim, Finland), equipped with a biphasic 70-mm F8 coil. Continuous TBS was delivered with MagPro stimulators. Electromyographic activity (EMG) was recorded with surface electrodes ( $\mathrm{Ag}-\mathrm{AgCl}, 10-\mathrm{mm}$ diameter) attached to the skin in a belly tendon montage over the right first dorsal interosseus (FDI) muscle (either with PowerLab 4/25T, AD Instruments Ltd., Australia, or with the NBS system). EMG activity was amplified and band-pass-filtered between 20 and $2000 \mathrm{~Hz}$, digitized (sample-rate $5 \mathrm{kHz}$ ) using specialized software (Scope $\mathrm{v} 4.0$ ), and stored for off-line analysis.

Subjects were seated in a comfortable chair with their elbows flexed at approximately $90^{\circ}$ and their hands resting while pronated on their laps. The optimal scalp location for activation of the right FDI using TMS was determined as the location from which TMSinduced motor evoked potentials (MEPs) of maximum peak-topeak amplitude were detected in the right FDI. The coil was moved in 1-cm increments over the scalp and we delivered single TMS pulses of constant supra-threshold stimulus intensity with an interstimulus interval (ISI) of at least $7 \mathrm{~s}$. The coil was consistently held tangentially to the scalp at an angle of approximately $45^{\circ}$ from the mid-sagittal plane with the handle pointing occipitally, such that the current induced in the brain flowed anterior to posterior, approximately perpendicular to the central sulcus. Once the optimal spot was identified, a marker was placed in a template magnetic resonance imaging (MRI) scan to which the individual subject was registered using a neuronavigational system, either Brainsight (Rogue Inc., Montreal, Canada) or the NBS system (in six subjects) to systematically place the TMS coil in the same location throughout the study. Six of our subjects, all from the Boston sample and those in which the NBS system was used, underwent a high-resolution structural MRI scan, which was also used to perform measurements of the distance from scalp to cortex at the hotspot/targeted brain region, and to assess possible cortical atrophy.

Motor threshold (MT) was determined according to the recommendations of the International Federation for Clinical Neurophysiology (Rossini et al., 1994). Single TMS pulses were delivered over the optimal scalp position for activation of the right FDI starting at supra-threshold intensity and gradually reduced by decrements of $2 \%$ of stimulator output. Resting MT (RMT) was defined as the lowest stimulus intensity capable of inducing MEPs of $\geq 50 \mu \mathrm{V}$ peak-to-peak amplitude in at least 5 of 10 consecutive trials. EMG monitoring was used to assure that the target muscle was at rest. Active MT (AMT) was then determined as the minimum single-pulse TMS intensity required to produce MEPs of $\geq 200 \mu \mathrm{V}$ in at least 5 of 10 consecutive trials while subjects contracted the target muscle (contralateral FDI) at approximately $20 \%$ of the maximal voluntary contraction.

Subsequently, we applied three batches of 10 single TMS pulses to the optimal FDI scalp position with ISI $\geq 7 \mathrm{~s}$ and intensity of $120 \%$ of RMT. Each batch was separated by a 5 -min pause. This procedure was then followed by a 40 -s train of cTBS (600 stimuli, three pulses at $50 \mathrm{~Hz}$ applied at a frequency of $5 \mathrm{~Hz}$ ), following the paradigm introduced by Huang et al. (2005), to the same scalp position. After CTBS, further batches of 10 single TMS pulses were acquired, at 5-, 10-, 20-, 30-, 40-, 50-, 60-, 75-, 90-, 105-, and 120min post-stimulation at the Boston site, and at 15, 30, 45, 60, 75, 90 , and $120 \mathrm{~min}$ at the Barcelona site. A schematic representation of study procedures is depicted in Figure 1A.

\section{STATISTICAL ANALYSIS}

All data analyses were conducted with MatLab (version 7.4.0). MEP amplitude at a given time-point was defined as the mean peakto-peak amplitude of the 10 MEPs to single TMS pulses recorded in a given batch. For each participant, baseline MEP amplitude was then defined as the average peak-to-peak amplitude of the 30 recorded MEPs. Mean MEP amplitude at each acquisition timepoint after cTBS was expressed as a proportion of baseline for each individual, defining an MEP threshold to represent the point at which post-cTBS MEP amplitude returned to the average MEP amplitude at baseline. Data were subsequently zeroed about the MEP threshold (i.e., MEP threshold was defined at zero on the dependent axis; Figure 1B).

Smooth curves through the data points were calculated using spline interpolation. Spline interpolation is a method of interpolation where the interpolant is a cubic spline, a piecewise continuous function defined by third-degree polynomials in the intervals of a 


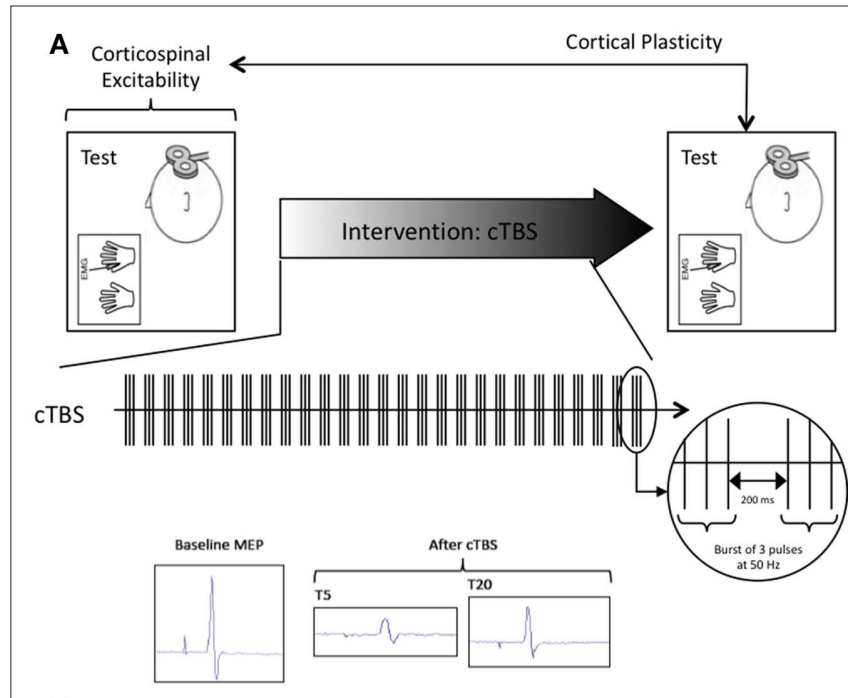

B

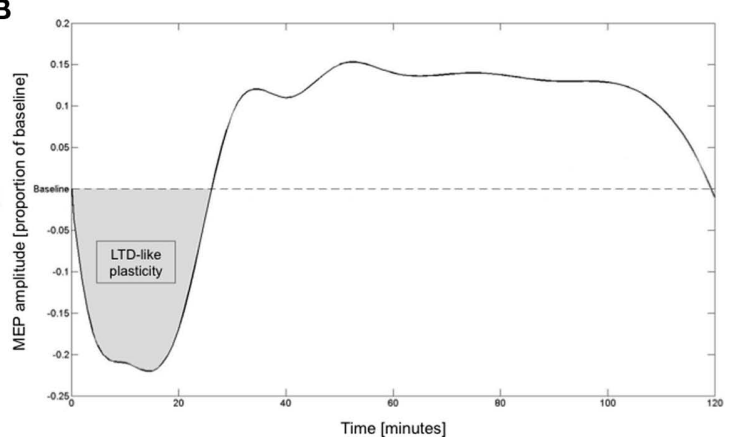

FIGURE 1 | (A) Schematic representation of study design; (B) Averaged spline for the combined sample plotted at each point in time assessed.

limited range of known data points (in this case, the time-points at which MEP data were collected with batches of 10 single TMS pulses). Spline interpolation has advantages over both linear and polynomial interpolation: It is more precise than linear interpolation and minimizes oscillation inaccuracies often found with high-degree polynomials. Spline interpolation has previously been described and successfully applied spatially to TMS data (Borghetti et al., 2008).

As an index of the duration of the TBS-induced modulation of cortico-spinal excitability, we defined, for each participant, the time-point ("time-to-baseline") at which post-cTBS MEP amplitude returned to the average MEP amplitude at baseline, i.e., the time-point at which the spline crossed the MEP threshold. In addition to time-to-baseline values, for each individual we calculated the area representing the amount of inhibition ("area of inhibition") following cTBS until return to baseline, i.e., the positive-valued area bounded below by the spline and above by each subject's MEP threshold. Finally, we compiled the minimum MEP amplitudes for each individual by computing the minimum point of the spline.

Individual data on each of the measures considered (i.e., MEP amplitude at baseline, times-to-baseline, area of inhibitory effect, and minimum MEP amplitude after cTBS) were averaged for each study site, and also averaged in a final sample combining all participants of both Boston and Barcelona sites. Differences in mean age, baseline MEP amplitude, time-to-baseline, area of inhibition, and post-cTBS minimum MEP amplitude between Boston and Barcelona samples were analyzed with unpaired two-sample $t$-tests. In order to test age-associated effects on MEP amplitude at baseline, times-to-baseline, area of inhibitory effect, and minimum MEP amplitude after cTBS in the combined sample, correlation coefficients between age and each of these variables were computed using Pearson's correlation. Statistical significance was defined at $p<0.05$.

\section{DISTANCE-TO-COIL MEASUREMENTS}

In a subset of subjects $(n=6)$, we performed measurements of the distance from the coil's location at the scalp to the cortical target as defined by the NBS system, in order to investigate whether potential changes in the cortical measures across the lifespan might be due to or influenced by brain atrophy with advancing age. For this purpose, the coordinates of each subject's hotspot and the TMS coil position, both given by the NBS system, were used to calculate the distance from scalp to brain. The hotspot was defined, at the cortical ribbon, at a depth of $25-\mathrm{mm}$ peeling view in the NBS system. This enabled the normalization of procedures for all subjects. To calculate the distance from the coil to the resulting hotspot coordinates we considered the applied direction of current (dependent on orientation of the TMS coil as recorded by the NBS). These coordinates were transformed and introduced into the Slicer software package (www.slicer.org) to estimate the point-to-point distance. Pearson correlation analyses between subjects' age, MEP amplitudes at baseline, times-to-baseline, areas of inhibitory effect, or minimum MEP amplitudes after cTBS, and the measurements of distance from coil to the hotspot were computed. Differences in age between this subgroup and the remaining subjects were analyzed with an independent, two-sample $t$-test.

\section{RESULTS}

\section{CHARACTERIZATION OF STUDY SAMPLES}

Mean age $( \pm S D)$ of the studied participants was $50.28 \pm 18.45$ years. All participants completed the study without unexpected events or side-effects of TMS or TBS. The study was conducted in parallel in Boston and in Barcelona. The samples and findings at each site were matched. Specifically, at the Boston site, the 14 subjects studied were on average $45.00 \pm 19.75$ years old, while at the Barcelona site the 22 individuals studied were $53.64 \pm 17.19$ years old. These age differences were not significant $(p=0.174)$. The average MEP peak-to-peak amplitude at the hotspot at baseline was $2.26 \pm 1.09 \mathrm{mV}$ for the Boston sample and $2.26 \pm 0.55 \mathrm{mV}$ for the Barcelona sample. There was no significant difference between sites on baseline MEP amplitude $(p=0.988)$. The average duration of the modulation of cortico-spinal excitability by cTBS, or time-to-baseline, was $26.80 \pm 5.72 \mathrm{~min}$ for the Boston sample and $28.88 \pm 7.50 \mathrm{~min}$ for the Barcelona sample. There was no statistically significant difference in time-to-baseline between study sites $(p=0.383)$. The cTBS-induced inhibition was also not statistically significant between the Boston and Barcelona sites $(p=0.579)$. For example, the area of inhibition was $5.07 \pm 2.30$ and $4.66 \pm 2.01$ in the Boston and Barcelona samples respectively. Finally, the average of the minimum amplitude of MEPs after cTBS was $-0.33 \pm 0.10$ and $-0.25 \pm 0.08 \mathrm{mV}$ for the Boston and Barcelona samples, respectively. This difference was, in contrast to all others, found to be 
statistically significant $(p=0.013$ ). Figure 1B depicts the averaged smooth curve throughout the time-points calculated using spline interpolation for the combined study sample.

\section{AGING-ASSOCIATED EFFECTS IN MOTOR CORTICAL PLASTICITY}

Average MEP peak-to-peak amplitude at the hotspot at baseline was $2.26 \pm 0.79 \mathrm{mV}$. There was a weak, non-significant correlation between age and baseline MEP amplitude $(r=-0.210 ; p=0.220)$. Following cTBS, mean time-to-baseline was $28.07 \pm 6.86 \mathrm{~min}$ (Figure 1B). This duration of the cTBS effect was linearly and inversely correlated with age $(r=-0.367 ; p=0.028)$. Figure 2A depicts these findings. Moreover, the mean area of inhibition after cTBS was $4.82 \pm 2.11$ (Figure 1B). Area of inhibition also showed as a significant inverse correlation with age, with the amount of inhibition after cTBS gradually reducing with advancing age $(r=-0.478 ; p=0.003)$. Figure 2B displays this correlation. Finally, the mean minimum MEP amplitude following cTBS was $-0.28 \pm 0.10 \mathrm{mV}$ and was significantly and positively correlated with age $(r=0.420 ; p=0.011)$, with older subjects showing smaller minimum MEP amplitude than their younger counterparts (Figure 2C).

\section{DISTANCE-TO-COIL EFFECTS IN MOTOR CORTICAL PLASTICITY FINDINGS}

The age-range and mean age of the subset of participants $(n=6$; 20-73 years, $48.17 \pm 22.52$ years) in whom we performed MRIbased anatomical measurements were representative of the overall sample. There was no significant difference between the ages of this subgroup and of the remaining participants $(n=30$, $50.70 \pm 17.95$ years; $p=0.764$ ). The average coil-to-brain distance was $26.93 \pm 0.39 \mathrm{~mm}$. As expected, we found an increase in coil-to-brain distance with advancing age, but this trend was not significant $(r=-0.774 ; p=0.071)$. Furthermore, there were no significant correlations between coil-to-brain distance and MEP amplitude at baseline $(r=-0.093 ; p=0.861)$, time-to-baseline after cTBS $(r=-0.143 ; p=0.788)$, area of inhibitory effect after cTBS $(r=-0.467 ; p=0.351)$, or minimum MEP amplitude after cTBS $(r=-0.163 ; p=0.758)$. Figure 3 displays the MRI-based anatomical measurement performed in one of the subjects.

\section{DISCUSSION}

In this cross-sectional study with subjects ranging from 19 to 81 years of age, we found that motor cortical plasticity, as induced by cTBS, progressively and linearly declines across the human lifespan. This effect was consistent across various measures of the cTBS effect. These findings suggest that LTD-like plasticity in the motor cortex is progressively reduced with aging.

Theta-burst stimulation has been shown to induce reliable and consistent cortical modulation that can be assessed by single-pulse TMS-induced MEPs (as in the present study), TMS-induced electroencephalographic (EEG) potentials or functional MRI (fMRI) responses (Huerta and Volpe, 2009). The modulatory effects of TBS on human local cortical reactivity are thought to mimic paradigms used to assess synaptic plasticity in animal models (Huang et al., 2005 , 2008). Although the neurobiological mechanisms of TBS in humans are not fully understood, a recent review concludes that this protocol modifies synaptic efficacy and induces LTP- and
LTD-like effects (Cárdenas-Morales et al., 2010). Whether such changes affect excitatory, inhibitory, or various types of synapses and at what cortical layers is unclear. Nevertheless, different patterns of TBS might modulate the activity of inhibitory cortical systems and protein expression differently, as suggested by animal studies (Benali et al., 2011). Additionally, in the motor cortex, these effects have been shown to reflect intracortical, rather than spinal, modulation of plasticity (Di Lazzaro et al., 2005). When TBS is applied continuously over the motor cortex - as it was in this study - the amplitude of TMS-induced MEPs following cTBS is reduced, consistent with an LTD-like form of plasticity (Huang et al., 2005). Increased GABAergic activity appears to underlie the effects of cTBS over the primary motor cortex (Stagg et al., 2009), consistent with the LTD dependency primarily on the GABAergic system (Hess and Donoghue, 1996).

Our findings confirm and extend recently reported findings by Todd et al. (2010) who found reduced motor cortex plasticity in older as compared to younger subjects in response to $10 \mathrm{~min}$ of 6-Hz rTMS at low intensity. The repetitive TMS (rTMS) protocol used by Todd et al. (2010) is thought to be less specific in the neurobiological substrates of the induced neuromodulation than TBS (Rossi et al., 2009). Furthermore, there is substantial inter- and even intra-individual variability in the modulation of cortico-spinal excitability by rTMS (Maeda et al., 2000), while the modulatory effects of TBS are more consistent within and across subjects. As compared with Todd et al. (2010), we studied a wider range of ages (19-81 years) and our paradigm enabled us to go beyond the comparison of two cohorts, and demonstrate a significant, inverse correlation with age.

We investigated the effects of cTBS, thought to capture LTD-like plasticity. A different TBS paradigm, intermittent or iTBS, allows the study of LTP-like plasticity (Huang et al., 2005), which may also undergo a progressive decline with advancing age. Another plasticity-inducing TMS paradigm, paired associative stimulation (PAS) - which involves the pairing of an electric stimulus to the peripheral median nerve with a TMS pulse over the contralateral motor cortex - has revealed an age-dependent reduction of LTPlike plasticity (Fathi et al., 2010). PAS captures heterotypic, sensory-motor spike timing-dependent plasticity (Stefan et al., 2000; Wolters et al., 2003). Future studies with iTBS might be interesting to assess homotypic, motor plasticity across aging.

The functional significance of the observed corticomotor plasticity decrement across the lifespan is unclear. Serial reaction time tasks may be useful in the investigation of the association between our neurophysiologic findings and the putative age-related deficits in sequence skill learning acquisition and consolidation (e.g., Brown et al., 2009). Indeed, Rogasch et al. (2009) recently examined training-induced changes in corticomotor excitability and plasticity in young and old adults, immediately and $30 \mathrm{~min}$ after a thumb abduction training task, and showed that MEP amplitudes became significantly larger in younger but not in older subjects, thus suggesting the existence of reduced use-dependent corticomotor plasticity with advancing age. In a more recent study, the same group showed an age-related decline in motor learning only in the dominant hand (Cirillo et al., 2010). Thus, future studies correlating TBS measures of cortical plasticity with behav- 

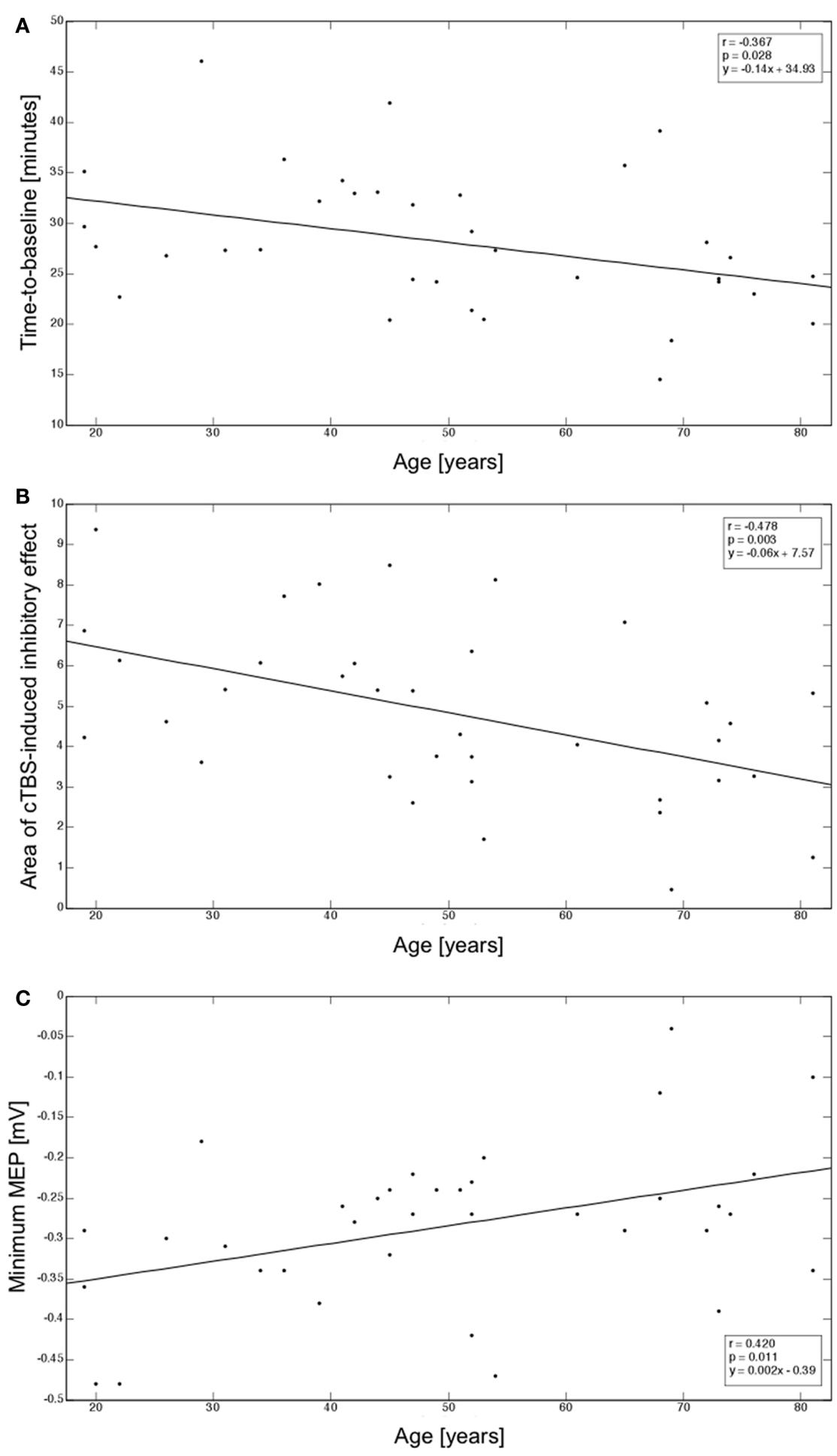

FIGURE 2 | (A) Correlation between age and time-to-baseline; (B) Correlation between age and area of inhibitory effects after cTBS; (C) Correlation between age and minimum MEP amplitude reached after cTBS. Correlation coefficients ( $h$ ), corresponding regression slopes, and intercept values are indicated (box).

ioral measures of motor learning are important. The progressive decrease in cTBS-induced plasticity we found in the motor cortex with advancing age may indeed be associated with age-dependent decline in hand motor function (e.g., longer reaction time) observed during healthy aging (e.g., Carmeli et al., 2003) and, especially, to age-related deficits in motor learning (e.g., Howard 


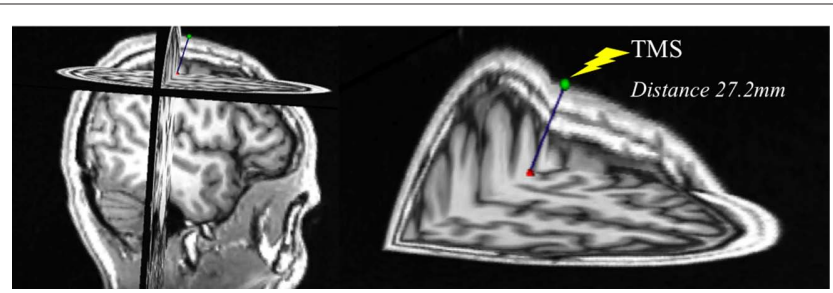

FIGURE 3 | Exemplification of the MRI-based measurement of distance from scalp to hotspot in a 22-year-old subject.

and Howard, 1989, 1992; Cherry and Stadler, 1995; Curran, 1997; Daselaar et al., 2003; Bunce et al., 2004; Brown et al., 2009). Agerelated compensatory mechanisms have been suggested based on functional neuroimaging evidence of differential age-related brain activation during motor (e.g., Mattay et al., 2002) and a variety of cognitive tasks (e.g., Resnick et al., 2007). Such compensatory mechanisms are typically characterized by over-recruitment of other brain regions (e.g., Cabeza et al., 2002), even when task difficulty is controlled for (Davis et al., 2008). It has been argued that such age increases in brain activity may, at least in part, be indicative of neural inefficiency (e.g., Grady, 2008). Indeed, there is recent evidence of increased activity on a subset of regions of the task-positive network (TPN) being associated with - and predictive of - worse performance (Grady et al., 2010), i.e., the greater the extent of the activity in the TPN in the older, the poorer the performance. This additional recruitment may still reflect an age-related compensatory mechanism, but may be the consequence of decreased neural efficiency in elders (Grady et al., 2010). Our results agree with such findings, and suggest that the progressive, insidious development of deficits in the mechanisms of neural plasticity may trigger, as a consequence, a cascade of adaptive or maladaptive responses resulting in a more or less salient decline of function.

Our findings of decreasing motor cortical plasticity with age may also be related to structural changes occurring during aging. The growth and stability of the dendritic arbor is highly regulated by intrinsic signals and extrinsic molecular mechanisms (Urbanska et al., 2008), namely neuronal activity-dependent effects (for review, Parrish et al., 2007). Additionally, AMPA (Haas et al., 2006) and NMDA (Sin et al., 2002) receptor-mediated glutamatergic and GABA-based (Gascon et al., 2006) neurotransmission may be involved in the dynamics of proper dendritic arborization. Altered plasticity mechanisms may, as a result, impair synaptic terminals and induce further remodeling of the dendritic arborization, eventually leading to structural abnormalities of brain tissue characterizing healthy aging and even psychiatric disorders (Penzes et al., 2011). Cross-sectional studies have consistently identified morphometric changes driven by healthy aging, typically encompassing regional cortical thinning, volumetric subcortical reductions, and ventricular enlargement (e.g., Walhovd et al., 2005, 2009; Fjell et al., 2009b). Longitudinal studies have demonstrated annual atrophy rates for brain volume, hippocampus, and entorhinal cortex (e.g., Scahill et al., 2003; Fotenos et al., 2005), and atrophy in cortical brain regions over different periods of time (Raz et al., 2005; Driscoll et al., 2009), often exceeding cross-sectional estimates (Raz et al., 2005). Notably, volumetric reductions of cortical and several subcortical brain regions, as well as ventricular expansion, have recently been shown after only 1 year in the healthy elderly (Fjell et al., 2009a) without subclinical cognitive disorders (Fjell et al., 2010). Motor cortex, in particular, undergoes prominent cortical thinning with age (Ziegler et al., 2008). Our findings may therefore provide a neurophysiologic correlate of the morphometric changes revealed by serial brain imaging studies across aging. Obviously, though, our cross-sectional findings warrant a follow-up longitudinal study to further characterize the results and explore inter-individual differences likely to be present.

It is important to examine whether our findings could be accounted for by age-related brain atrophy or an increase of coilto-hotspot distance. It is known that the impact of TMS, and thus also TBS, depends on the distance between cortex and scalp as the magnetic field, and thus also the induced electric field, decreases with distance (Wagner et al., 2004, 2008). Furthermore, modeling work suggests that current density distribution is critically influenced by brain morphology and tissue characteristics (Wagner et al., 2007, 2008). Brain atrophy can thus substantially alter the impact of TMS (Wagner et al., 2007) not only because of the effect of greater scalp-to-brain distance, but also due to increased current shunting in the cerebrospinal fluid (CSF) compartment. Therefore, it is relevant to consider the possibility that age-related differences in TMS or TBS effects might simply reflect cortical atrophy. We did find a trend for an increased coil-to-brain distance with advancing age in the subset of subjects analyzed, and it is possible that we could have found significant results if similar measurements had been performed in the total number of participants (greater power). Nevertheless, our data make the possibility of a major influence of brain atrophy on our neurophysiologic results very unlikely. First, baseline MT and MEP amplitudes showed no significant correlation with age. Second, correlations between coilto-brain distance and MEP at baseline, duration of TBS effect, area of inhibitory effect, or minimum MEP after cTBS were all weak and non-significant. Therefore, while we cannot completely rule out a possible contribution of atrophy, our findings seem primarily related to age-associated modifications of the physiological processes measured by TMS rather than anatomical differences. Nevertheless, future studies fully accounting for this potential confound are warranted.

Finally, it is worth noting that statistical analyses revealed that samples from Boston and Barcelona behaved similarly, with congruent effects obtained across study sites in regard to baseline MEPs and modulation after cTBS for all measures, except for the minimum MEP amplitude reached after cTBS. The significance of the differences in this one measure is unclear. Importantly, all other measures are consistent and all show the same impact of aging, for the Boston and Barcelona samples separately and combined.

\section{CONCLUSION}

Our cross-sectional results demonstrate a linear decline of human motor cortical plasticity during healthy aging. We focused our study on the motor cortex, but predict that similar changes in the mechanisms of plasticity may be found in non-motor, 
associative neocortical areas underlying cognitive decline. Our paradigm enables the study of plasticity mechanisms in nonmotor cortical brain regions if combined with EEG measures in a real-time integrated approach (Thut and Pascual-Leone, 2010). The presented measures, in the setting of longitudinal studies, may allow for the assessment of the impact of environmental and biological factors (e.g., diet, physical activity, chronic stress, sleep deprivation, stroke, traumatic brain injury, hypertension, diabetes or menopause) on age-associated changes in plasticity. Furthermore, such measures of cortical plasticity may serve as biomarkers to guide plasticity-based interventions aimed at promoting healthy aging and cognitive well-being across the age-span. Such interventions may include non-invasive brain stimulation approaches, as recently proposed by Zimerman and Hummel (2010).

\section{REFERENCES}

Andrews-Hanna, J. R., Snyder, A. Z., Vincent, J. L., Lustig, C., Head, D., Raichle, M. E., and Buckner, R. L. (2007). Disruption of large-scale brain systems in advanced aging. Neuron 56 , 924-935.

Barnes, C. A. (1979). Memory deficits associated with senescence: a neurophysiological and behavioral study in the rat. J. Comp. Physiol. Psychol. 93, 74-104.

Barnes, C. A., and McNaughton, B. L. (1980). Physiological compensation for loss of afferent synapses in rat hippocampal granule cells during senescence. J. Physiol. 309, 473-485.

Benali, A., Trippe, J., Weiler, E., Mix, A., Petrasch-Parwez, E., Girzalsky, W., Eysel, U. T., Erdmann, R., and Funke, K. (2011). Theta-burst transcranial magnetic stimulation alters cortical inhibition. J. Neurosci. 31, 1193-1203.

Bliss, T. V., Collingridge, G. L., and Morris, R. G. (2003). Introduction. Long-term potentiation and structure of the issue. Philos. Trans. R. Soc. Lond. B Biol. Sci. 358, 607-611.

Borghetti, D., Sartucci, F., Petacchi, E., Guzzetta,A., Piras, M. F., Murri, L., and Cioni, G. (2008). Transcranial magnetic stimulation mapping: a model based on spline interpolation. Brain Res. Bull. 77, 143-148.

Brown, R.M., Robertson, E. M., and Press, D.Z. (2009). Sequence skill acquisition and off-line learning in normal aging. PLoS ONE4, e6683. doi: 10.1371/journal.pone.0006683

Bunce, D., MacDonald, S. W., and Hultsch, D. F. (2004). Inconsistency in serial choice decision and motor reaction times dissociate in younger and older adults. Brain Cogn. 56, 320-327.

Cabeza, R., Anderson, N. D., Locantore, J. K., and McIntosh, A. R. (2002). Aging gracefully: compensatory brain activity in high-performing older adults. Neuroimage 17, 1394-1402.
Cárdenas-Morales, L., Nowak, D. A., Kammer, T., Wolf, R. C., and Schönfeldt-Lecuona, C. (2010). Mechanisms and applications of thetaburst rTMS on the human motor cortex. Brain Topogr. 22, 294-306.

Carmeli, E., Patish, H., and Coleman, R. (2003).The aging hand. J. Gerontol. A Biol. Sci. Med. Sci. 58, 146-152.

Cherry, K. E., and Stadler, M. A. (1995). Implicit learning of a nonverbal sequence in younger and older adults. Psychol. Aging 10, 379-394.

Cirillo, J., Rogasch, N. C., and Semmler, J. G. (2010). Hemispheric differences in use-dependent corticomotor plasticity in young and old adults. Exp. Brain Res. 205, 57-68.

Curran, T. (1997). Effects of aging on implicit sequence learning: accounting for sequence structure and explicit knowledge. Psychol. Res. 60, 24-41.

Damoiseaux, J. S., Beckmann, C. F., Arigita, E. J., Barkhof, F., Scheltens, P., Stam, C. J., Smith, S. M., and Rombouts, S. A. (2008). Reduced resting-state brain activity in the "default network" in normal aging. Cereb. Cortex 18, 1856-1864.

Daselaar, S. M., Rombouts, S.A., Veltman, D. J., Raaijmakers, J. G., and Jonker, C. (2003). Similar network activated by young and old adults during the acquisition of a motor sequence. Neurobiol. Aging 24, 1013-1019.

Davis, S. W., Dennis, N. A., Daselaar, S. M., Fleck, M. S., and Cabeza, R. (2008). Que PASA? The posterioranterior shift in aging. Cereb. Cortex 18, 1201-1209.

Deary, I. J., Corley, J., Gow, A. J., Harris, S. E., Houlihan, L. M., Marioni, R. E., Penke, L., Rafnsson, S. B., and Starr, J. M. (2009). Age-associated cognitive decline. Br. Med. Bull. 92, 135-152.

Di Lazzaro, V., Pilato, F., Saturno, E., Oliviero, A., Dileone, M., Mazzone, P., Insola, A., Tonali, P. A., Ranieri, F., Huang, Y. Z., and Rothwell, J. C.

\section{ACKNOWLEDGMENTS}

Work on this study was supported by grants from the National Center for Research Resources: Harvard-Thorndike General Clinical Research Center at BIDMC (NCRR MO1 RR01032) and Harvard Clinical and Translational Science Center (UL1 RR025758), NIH grant K24 RR018875, and a grant from the Nancy Lurie Marks Family Foundation to Alvaro Pascual-Leone. Catarina Freitas was supported by post-doctoral grants from the Foundation for Science and Technology, Portugal (SFRH/BPD/44126/2008 and SFRH/ BPD/66846/2009), co-funded by the European Social Fund. Lindsay Oberman was supported by NIH fellowship F32MH080493 and 1KL2RR025757-01. Jose M. Tormos was supported by Foundation La Marató TV3 (071931) and grant PI082004 from the Instituto de Salud Carlos III. Marine Vernet was supported by the Fyssen Foundation, France.

(2005). Theta-burst repetitive transcranial magnetic stimulation suppresses specific excitatory circuits in the human motor cortex. J. Physiol. 565(Pt 3), 945-950.

Driscoll, I., Davatzikos, C., An, Y., Wu, X., Shen, D., Kraut, M., and Resnick, S. (2009). Longitudinal pattern of regional brain volume change differentiates normal aging from MCI. Neurology 72, 1906-1913.

Fathi, D., Ueki, Y., Mima, T., Koganemaru, S., Nagamine, T., Tawfik, A., and Fukuyama, H. (2010). Effects of aging on the human motor cortical plasticity studied by paired associative stimulation. Clin. Neurophysiol. 121, 90-93.

Feier, C. D., and Gerstman, L. J. (1980). Sentence comprehension abilities throughout the adult life span. $J$. Gerontol. 35, 722-728.

Fjell, A. M., Walhovd, K. B., FennemaNotestine, C., McEvoy, L. K., Hagler, D. J., Holland, D., Brewer, J. B., and Dale, A. M. (2009a). One-year brain atrophy evident in healthy aging. $J$. Neurosci. 29, 15223-15231.

Fjell, A. M., Westlye, L. T., Amlien, I. Espeseth, T., Reinvang, I., Raz, N., Agartz, I., Salat, D. H., Greve, D. N., Fischl, B., Dale, A. M., and Walhovd, K. B. (2009b). High consistency of regional cortical thinning in aging across multiple samples. Cereb. Cortex 19, 2001-2012.

Fjell, A. M., Westlye, L. T., Espeseth, T. Reinvang, I., Dale, A. M., Holland, D., and Walhovd, K. B. (2010). Cortical gray matter atrophy in healthy aging cannot be explained by undetected incipient cognitive disorders: a comment on Burgmans et al. (2009). Neuropsychology 24, 258-263. [Discussion 264-266].

Folstein, M. F., Folstein, S. E., and McHugh, P. R. (1975). "Mini-mental state”. A practical method for grading the cognitive state of patients for the clinician. J. Psychiatr. Res. 12, 189-198.
Fotenos, A. F., Snyder, A. Z., Girton, L. E., Morris, J.C., and Buckner, R. L. (2005). Normative estimates of cross-sectional and longitudinal brain volume decline in aging and AD. Neurology 64 , 1032-1039.

Gascon, E., Dayer, A. G., Sauvain, M. O., Potter, G., Jenny, B., De Roo, M., Zgraggen, E., Demaurex, N., Muller, D., and Kiss, J. Z. (2006). GABA regulates dendritic growth by stabilizing lamellipodia in newly generated interneurons of the olfactory bulb. J. Neurosci. 26, 12956-12966.

Grady, C. L. (2008). Cognitive neuroscience of aging. Ann. N. Y. Acad. Sci. 1124, 127-144.

Grady, C. L., Protzner, A. B., Kovacevic, N., Strother, S. C., Afshin-Pour, B., Wojtowicz,M.,Anderson,J.A., Churchill, N., and McIntosh,A.R. (2010).A multivariate analysis of age-related differences in default mode and task-positive networks across multiple cognitive domains. Cereb. Cortex 20, 1432-1447.

Haas, K., Li, J., and Cline, H. T. (2006). AMPA receptors regulate experiencedependent dendritic arbor growth in vivo. Proc. Natl. Acad. Sci. U.S.A 103, 12127-12131.

Hess, G., and Donoghue, J. P. (1996). Long-term depression of horizontal connections in rat motor cortex. Eur. J. Neurosci. 8, 658-665.

Howard, D. V., and Howard, J. H. Jr. (1989). Age differences in learning serial patterns: direct versus indirect measures. Psychol. Aging 4, 357-364.

Howard, D. V., and Howard, J. H. Jr. (1992). Adult age differences in the rate of learning serial patterns: evidence from direct and indirect tests. Psychol. Aging 7, 232-241.

Huang, Y. Z., Edwards, M. J., Rounis, E., Bhatia, K.P., and Rothwell, J.C. (2005). Theta burst stimulation of the human motor cortex. Neuron 45, 201-206.

Huang, Y. Z., Rothwell, J. C., Edwards, M. J., and Chen, R. S. (2008). Effect of 
physiological activity on an NMDAdependent form of cortical plasticity in human. Cereb. Cortex 18, 563-570.

Huerta, P. T., and Volpe, B. T. (2009). Transcranial magnetic stimulation, synaptic plasticity and network oscillations. J. Neuroeng. Rehabil. 6, 7.

Kelly, K. M., Nadon, N. L., Morrison, J. H., Thibault, O., Barnes, C. A., and Blalock, E. M. (2006). The neurobiology of aging. Epilepsy Res. 68(Suppl 1), $S 5-S 20$

Larson, J., Wong, D., and Lynch, G. (1986). Patterned stimulation at the theta frequency is optimal for the induction of hippocampal long-term potentiation. Brain Res. 368, 347-350.

Lutz, W., Sanderson, W., and Scherbov, S. (2008). The coming acceleration of global population ageing. Nature 451, 716-719.

Maeda, F., Keenan, J. P., Tormos, J. M., Topka, H., and Pascual-Leone, A. (2000). Interindividual variability of the modulatory effects of repetitive transcranial magnetic stimulation on cortical excitability. Exp. Brain Res. $133,425-430$.

Mattay, V. S., Fera, F., Tessitore, A., Hariri, A. R., Das, S., Callicott, J. H., and Weinberger, D. R. (2002). Neurophysiological correlates of agerelated changes in human motor function. Neurology 58, 630-635.

Oberman, L. M., Ifert-Miller, F., Najib, U., Bashir, S., Woollacott, I., GonzalezHeydrich, J., Picker, J., Rotenberg, A., and Pascual-Leone, A. (2010). Transcranial magnetic stimulation provides a means to assess cortical plasticity and excitability in humans with fragile $\mathrm{X}$ syndrome and autism spectrum disorders. Front. Synaptic Neurosci. 2:26. doi: $10.3389 /$ fnsyn. 2010.00026

Oldfield, R. C. (1971). The assessment and analysis of handedness: the Edinburgh inventory. Neuropsychologia 9, 97-113.

Parrish, J. Z., Emoto, K., Kim, M. D., and Jan, Y. N. (2007). Mechanisms that regulate establishment, maintenance, and remodeling of dendritic fields. Annu. Rev. Neurosci. 30, 399-423.

Penzes, P., Cahill, M. E., Jones, K. A., VanLeeuwen, J.-E., and Woolfrey, K. M. (2011). Dendritic spine pathology in neuropsychiatric disorders. Nat. Neurosci. 14, 285-293.

Raz, N.,Lindenberger, U., Rodrigue, K.M., Kennedy, K.M., Head, D., Williamson,
A., Dahle, C., Gerstorf, D., and Acker, J.D. (2005). Regional brain changes in aging healthy adults: general trends, individual differences and modifiers. Cereb. Cortex 15, 1676-1689.

Resnick, S. M., Lamar, M., and Driscoll, I. (2007). Vulnerability of the orbitofrontal cortex to age-associated structural and functional brain changes. Ann. N. Y. Acad. Sci. 1121, 562-575.

Rogasch, N. C., Dartnall, T. J., Cirillo, J., Nordstrom, M. A., and Semmler, J. G. (2009). Corticomotor plasticity and learning of a ballistic thumb training task are diminished in older adults. J. Appl. Physiol. 107, 1874-1883.

Roman, F., Staubli, U., and Lynch, G. (1987). Evidence for synaptic potentiation in a cortical network during learning. Brain Res. 418, 221-226.

Rosenzweig, E. S., and Barnes, C. A. (2003). Impact of aging on hippocampal function: plasticity, network dynamics, and cognition. Prog. Neurobiol. 69, 143-179.

Rossi, S., Hallett, M., Rossini, P. M., Pascual-Leone, A., and Safety of TMS Consensus Group. (2009). Safety, ethical considerations, and application guidelines for the use of transcranial magnetic stimulation in clinical practice and research. Clin. Neurophysiol. 120, 2008-2039.

Rossini, P. M., Barker, A. T., Berardelli, A., Caramia, M. D., Caruso, G., Cracco, R. Q., Dimitrijević, M. R., Hallett, M., Katayama, Y., Lücking, C. H., Maertens de Noordhout, A. L., Marsden, C. D., Murray, N. M. F., Rothwell, J. C., Swash, M., and Tomberg, C. (1994). Non-invasive electrical and magnetic stimulation of the brain, spinal cord and roots: basic principles and procedures for routine clinical application. Report of an IFCN committee. Electroencephalogr. Clin. Neurophysiol. 91, 79-92.

Sambataro, F., Murty, V. P., Callicott, J. H., Tan, H. Y., Das, S., Weinberger, D. R., and Mattay, V. S. (2010). Age-related alterations in default mode network: impact on working memory performance. Neurobiol. Aging 31, 839-852.

Scahill, R. I., Frost, C., Jenkins, R., Whitwell, J. L., Rossor, M. N., and Fox, N. C. (2003). A longitudinal study of brain volume changes in normal aging using serial registered magnetic resonance imaging. Arch. Neurol. 60, 989-994.
Sin, W. C., Haas, K., Ruthazer, E. S., and Cline, H. T. (2002). Dendrite growth increased by visual activity requires NMDA receptor and Rho GTPases. Nature 419, 475-480.

Stagg, C. J., Wylezinska, M., Matthews, P. M., Johansen-Berg, H., Jezzard, P., Rothwell, J. C., and Bestmann, S. (2009). Neurochemical effects of theta burst stimulation as assessed by magnetic resonance spectroscopy. $J$. Neurophysiol. 101, 2872-2877.

Stefan, K., Kunesch, E., Cohen, L. G., Benecke, R., and Classen, J. (2000). Induction of plasticity in the human motor cortex by paired associative stimulation. Brain 123(Pt 3), 572-584.

Thut, G., and Pascual-Leone, A. (2010). Integrating TMS with EEG: How and what for? Brain Topogr. 22, 215-218.

Todd, G., Kimber, T. E., Ridding, M. C., and Semmler, J. G. (2010). Reduced motor cortex plasticity following inhibitory rTMS in older adults. Clin. Neurophysiol. 121, 441-447.

Urbanska, M., Blazejczyk, M., and Jaworski, J. (2008). Molecular basis of dendritic arborization. Acta Neurobiol Exp. (Wars) 68, 264-288.

Wagner, T., Eden, U., Fregni, F., ValeroCabre, A., Ramos-Estebanez, C., Pronio-Stelluto, V., Grodzinsky, A., Zahn, M., and Pascual-Leone, A (2008). Transcranial magnetic stimulation and brain atrophy: a computerbased human brain model study. Exp. Brain Res. 186, 539-550.

Wagner, T., Gangitano, M., Romero, R., Théoret, H., Kobayashi, M., Anschel, D., Ives, J., Cuffin, N., Schomer, D., and Pascual-Leone, A. (2004). Intracranial measurement of current densities induced by transcranial magnetic stimulation in the human brain. Neurosci. Lett. 354, 91-94.

Wagner, T., Valero-Cabre, A., and PascualLeone, A. (2007). Noninvasive human brain stimulation. Annu. Rev. Biomed Eng. 9, 527-565.

Walhovd, K. B., Fjell, A. M., Reinvang, I., Lundervold, A., Dale, A. M., Eilertsen, D. E., Quinn, B. T., Salat, D., Makris, N., and Fischl, B. (2005). Effects of age on volumes of cortex, white matter and subcortical structures. Neurobiol. Aging 26, 1261-1270.

Walhovd, K. B., Westlye, L. T., Amlien, I., Espeseth, T., Reinvang, I., Raz,
N., Agartz, I., Salat, D. H., Greve, D. N., Fischl, B., Dale, A. M., and Fjell, A. M. (2009). Consistent neuroanatomical age-related volume differences across multiple samples. Neurobiol. Aging. doi: 10.1016/j.neurobiolaging. 2009.05.013

Wang, L., Li, Y., Metzak, P., He, Y., and Woodward, T. S. (2010). Age-related changes in topological patterns of large-scale brain functional networks during memory encoding and recognition. Neuroimage 50, 862-872.

Wolters, A., Sandbrink, F., Schlottmann, A., Kunesch, E., Stefan, K., Cohen, L. G., Benecke, R., and Classen, J. (2003). A temporally asymmetric Hebbian rule governing plasticity in the human motor cortex. J. Neurophysiol. 89, 2339-2345.

Ziegler, D. A., Piguet, O., Salat, D. H., Prince, K., Connally, E., and Corkin, S. (2008). Cognition in healthy aging is related to regional white matter integrity, but not cortical thickness. Neurobiol. Aging. 31, 1912-1926.

Zimerman, M., and Hummel, F.C. (2010). Non-invasive brain stimulation: enhancing motor and cognitive functions in healthy old subjects. Front. Aging Neurosci. 2:149. doi: 10.3389/ fnagi.2010.00149

Conflict of Interest Statement: The authors declare that the research was conducted in the absence of any commercial or financial relationships that could be construed as a potential conflict of interest.

Received: 26 February 2011; accepted: 23 March 2011; published online: 09 April 2011.

Citation: Freitas C, Perez J, Knobel M, Tormos JM, Oberman L, Eldaief M, Bashir $S$, Vernet $M$, Peña-Gómez $C$ and PascualLeone A (2011) Changes in cortical plasticity across the lifespan. Front. Ag. Neurosci. 3:5. doi: 10.3389/fnagi.2011.00005 Copyright $\odot 2011$ Freitas, Perez, Knobel, Tormos, Oberman, Eldaief, Bashir, Vernet, Peña-Gómez and Pascual-Leone. This is an open-access article subject to a nonexclusive license between the authors and Frontiers Media SA, which permits use, distribution and reproduction in other forums, provided the original authors and source are credited and other Frontiers conditions are complied with. 